\title{
Efecto Doppler con una fuente que acelera: una simple aproximación gráfica
}

\author{
Doppler effect with a source that accelerates: a simple graphical approach
}

\author{
C.H. Wörner*1, R. Rojas ${ }^{2}$ \\ ${ }^{1}$ Programa de Magister en Didáctica de las Ciencias Experimentales, Pontificia Universidad Católica de Valparaíso, \\ Valparaíso, Chile \\ ${ }^{2}$ Departamento de Física, Universidad Técnica Federico Santa María, Vitacura, Santiago, Chile
}

\begin{abstract}
Recebido em 30 de março de 2016. Revisado em 13 de junho de 2016. Aceito em 20 de julho de 2016
Se analiza el efecto Doppler producido por una fuente acelerada usando un enfoque cinemático elemental. Con este simple método se obtienen fácilmente las expresiones para la frecuencia detectada por un observador estacionario y una fuente que se aleja o acerca. También se analiza el caso relativista. Palabras clave: efecto Doppler, diagramas $(\mathrm{x}, \mathrm{t})$, cinemática.
\end{abstract}

The classical Doppler effect produced by an accelerated source is considered by using a naive kinematical, graphical approach. We obtain expressions for the frecuency detected by a stationary observer from a source that moves towards or away from him. An extension to the relativistic case is also analysed.

Keywords: Doppler effect, (x-t) diagrams, kinematics.

\section{Introducción}

En el tratamiento introductorio habitual a la física, el estudio del efecto Doppler clásico se describe en el capítulo que se ocupa de fenómenos ondulatorios. Así, este tema se introduce después de la descripción de los diversos fenómenos de ondas, y por lo tanto aparece ante los estudiantes como un tema complejo.

Esta cuestión ya se ha tratado-con variados maticesen diversos estudios que parecen haber sido pasados por alto en la práctica docente actual [1-5]. Recientemente apareció una nota sobre el retraso relativista de los relojes, utilizando un enfoque similar [6]. Un caso de corrimiento Doppler causado por el movimiento acelerado (de hecho, la caída libre) se propone como un ejercicio en el conocido libro de Serway [7].

El propósito de esta nota es aplicar un método gráfico, para resolver una cuestión que no suele tratarse en la práctica habitual de la enseñanza del efecto Doppler. Esto es, el caso en que la fuente y/o el observador están acelerando. Por simplici-

*Endereço de correspondência: cworner@ucv.cl dad, analizaremos el caso en que la fuente describe un movimiento rectilíneo uniformemente acelerado. Con respecto al caso en que el observador describe un movimiento acelerado, sólo haremos un comentario sobre su resultado. Este análisis es válido para ondas periódicas, que pueden ser pulsos o armónicas, propagándose en un medio homogéneo, de carácter elástico o electromagnético.

\section{El caso unidimensional con aceleración constante}

Consideremos una fuente que emite con frecuencia $(f=1 / T)$ y se mueve con aceleración constante en una trayectoria rectilínea (eje $\mathrm{X}$ ), respecto a un sistema de referencia inercial. Un observador en reposo en $\mathrm{x}=\mathrm{x}_{0}$ percibe una frecuencia $\left(1 / T^{\prime}\right) \mathrm{si}$ la fuente se aproxima y $\left(1 / T^{\prime \prime}\right)$ si ésta se aleja. El diagrama posición-tiempo, $(\mathrm{x}, \mathrm{t})$, de esta situación se puede ver en la Fig. 1; allí el gráfico que representa el movimiento de la fuente, de acuerdo a la conocida dependencia cuadrática de la posición con el tiempo, es una parábola. Supondremos que la posición y 
velocidad inicial, en $\mathrm{t}=0$ son: $\mathrm{x}(0)=0$ y $\mathrm{v}(0)=$ $V_{E}$.

Consideremos que en cierto instante $t$ se inicia la emisión de señales de período $T$. Mediante dos señales sucesivas y un análisis simple de las áreas $I$ y $I I$ de la Fig. 1, es posible relacionar los periodos $T$ y $T^{\prime}$ (o $\left.T^{\prime \prime}\right)$. Esta situación ampliada aparece en la Fig. 2. En el triángulo $I$ uno de los catetos es $T-T^{\prime}$ y - usando las conocidas relaciones para el movimiento uniformemente acelerado con velocidad inicial $V_{E}-$ el otro cateto resulta $V_{E} T+\left(t+\frac{T}{2}\right) a T$, siendo $a$ la aceleración constante del emisor. Dado que la pendiente de la hipotenusa corresponde a la velocidad de propagación de la onda $(V)$ el cociente entre los catetos debe satisfacer la siguiente relación:

$$
V=\frac{V_{E} T+\left(t+\frac{T}{2}\right) a T}{T-T^{\prime}} .
$$

Resolviendo para $T^{\prime}$ resulta:

$$
T^{\prime}=\frac{V-V_{E}-a t-a T / 2}{V} T,
$$

y la frecuencia es

$$
\begin{aligned}
& f^{\prime}=\frac{1}{1-\frac{V_{E}}{V}-\frac{a t}{V}-\frac{a}{2 f V}} f ; \\
& a t<\sqrt{2 a x_{0}+V_{E}^{2}}-V_{E}
\end{aligned}
$$
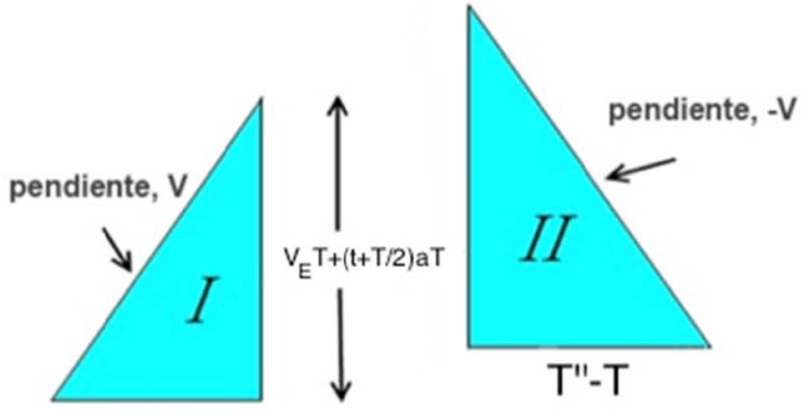

$\mathrm{T}-\mathrm{T}^{\prime}$

Figura 2: Ampliación de los triángulos marcados en la Fig. 1.

De manera análoga, la consideración del triángulo II, nos permite escribir:

$$
T^{\prime \prime}=\frac{V+V_{E}+a t+a T / 2}{V} T,
$$

y la frecuencia es

$$
\begin{aligned}
& f^{\prime \prime}=\frac{1}{1+\frac{V_{E}}{V}+\frac{a t}{V}+\frac{a}{2 f V}} f ; \\
& a t>\sqrt{2 a x_{0}+V_{E}^{2}}-V_{E}
\end{aligned}
$$

Estas expresiones no son desconocidas en la literatura científica, aunque son muy poco mencionadas.

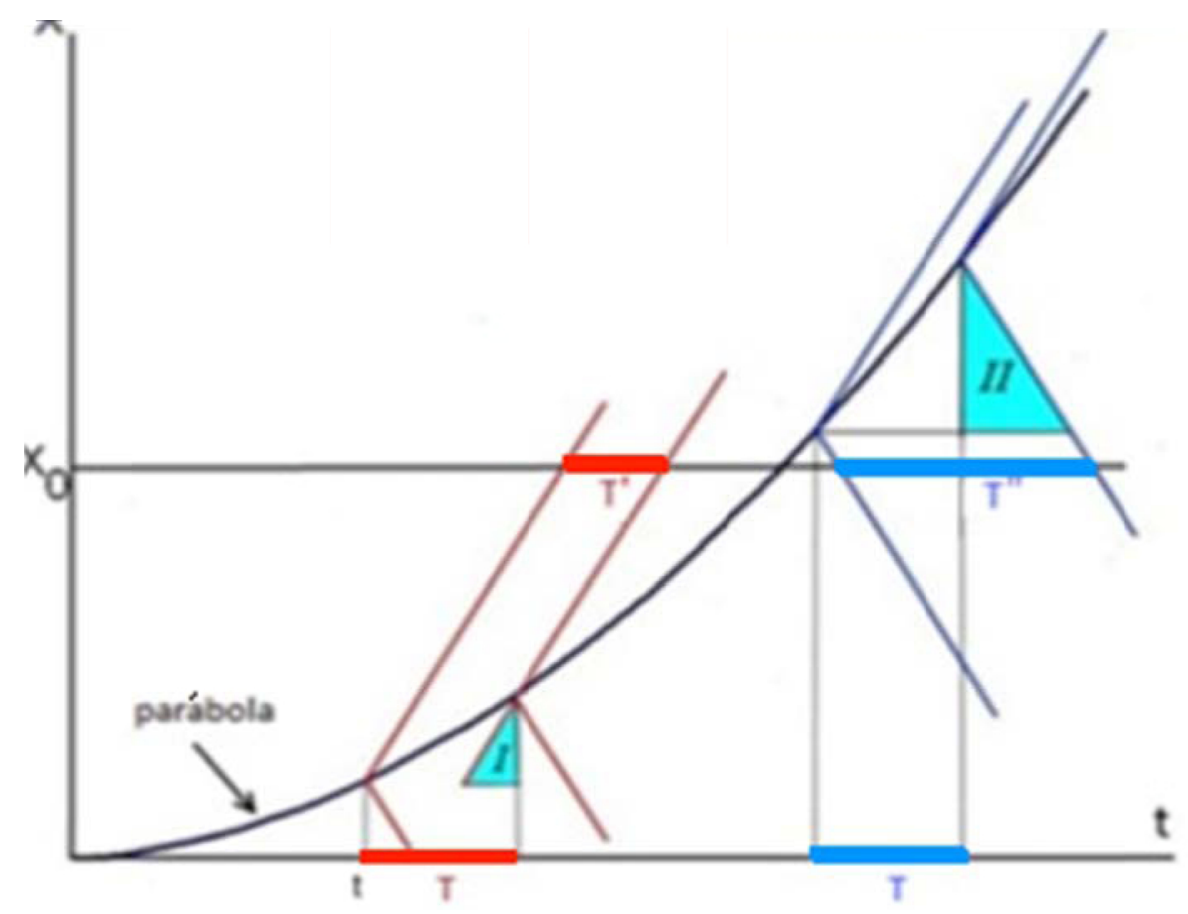

Figura 1: Diagrama $(x, t)$ para el caso de una fuente acelerando uniformemente y emitiendo con período $T$. Se observan las señales que percibe un observador en reposo, al acercarse o alejarse la fuente. 
En ellas, la velocidad del emisor no puede exceder la velocidad de propagación de las ondas, es decir, su validez está limitada al intervalo de tiempo en que se cumple: $0<a t<V-V_{E}$. El caso particular de aceleración nula proporciona resultados bien conocidos en los textos de introducción a la física. Radovic trata este caso con aceleración en una revista poco conocida, [8] obteniendo los mismos resultados a partir de un desarrollo en serie de la frecuencia percibida. En un interesante artículo, Neipp et al. [9] tratan el efecto Doppler clásico en tres dimensiones, y entre los diversos casos que analizan, obtienen una expresión para el caso de un observador que se mueve con una aceleración constante, alejándose radialmente desde la fuente. Su resultado (30) indica que la frecuencia disminuye linealmente en el tiempo, lo cual coincide con nuestro análisis del caso de un observador en movimiento acelerado cuando hacemos la aproximación at $<<V$. El método gráfico que hemos expuesto anteriormente se aplica de la misma manera al caso de un observador en movimiento acelerado y conduce a una ecuación de segundo grado que debe resolverse para obtener los periodos $T^{\prime}$ y $T^{\prime \prime}$.

\section{Algunos comentarios relativistas}

La extensión de este tratamiento para el caso relativista sin aceleración ha sido ya discutido en la literatura [4]. Earman y Glymour [10] han considerado la dificultad de introducir el concepto de aceleración en el marco de la relatividad. El problema se deriva de la idea original de Einstein, y su concepción inicial fue posteriormente re-articulada con las observaciones de Minskowski y Born (ver el citado trabajo de Earman y Glymour para seguir la discusión conceptual).

En nuestro caso, y de acuerdo con el resultado conocido como dilatación del tiempo, parece atractivo extender las Ec. (1) y (2) con sólo sustituir $T \rightarrow \gamma T$; siendo $\gamma$ el coeficiente:

$$
\gamma=\frac{1}{\sqrt{1-\left(\frac{a t}{V}\right)^{2}}}
$$

De esta manera, para un observador en reposo en un sistema de referencia inercial y una fuente en movimiento rectilíneo acelerado según $x(t)=$ $\frac{1}{2} a t^{2}$, obtenemos los resultados siguientes para los casos en que ésta se acerca o se aleja del observador respectivamente:

$$
\begin{aligned}
& T^{\prime}=\frac{V-a t-\frac{a \gamma T}{2}}{V}(\gamma T), \\
& T^{\prime \prime}=\frac{V+a t+\frac{a \gamma T}{2}}{V}(\gamma T) .
\end{aligned}
$$

De hecho, este ansatz es una expresión aproximada para el caso que nos ocupa como veremos a continuación

Consideremos dos sistemas de referencia S y S', con coordenadas respectivas $(\mathrm{x}, \mathrm{t}) \mathrm{y}\left(\mathrm{x}^{\prime}, \mathrm{t}^{\prime}\right)$, este último viajando con velocidad $V_{R}=a t$ en la dirección $+\mathrm{X}$ respecto al sistema inercial $\mathrm{S}$. En el origen del sistema $\mathrm{S}$ ' se emiten pulsos periódicos que se propagan con rapidez $V$ respecto a $\mathrm{S}$, dos de los pulsos se emiten en los instantes $\mathrm{t}_{1}$ ' y $\mathrm{t}_{2}$ ' cuyo período en el emisor S' es definido por $\tau_{0}=t_{2}^{\prime}-t_{1}^{\prime}$ . En el sistema $\mathrm{S}$ las coordenadas $(\mathrm{x}, \mathrm{t})$ de estos eventos de emisión son $\left(x_{1}, t_{1}\right)$ y $\left(x_{2}, t_{2}\right)$ respectivamente, siendo $x_{1}=\frac{1}{2} a t_{1}^{2}$ y $x_{2}=\frac{1}{2} a t_{2}^{2}$. Los pulsos son recibidos en el origen del sistema de referencia $\mathrm{S}$ del observador en los instantes $T_{1}^{*}=t_{1}+x_{1} / V$ y $T_{2}^{*}=t_{2}+x_{2} / V$, lo que permite definir el período en el receptor como $\tau=T_{2}^{*}-T_{1}^{*}$ que satisface la ecuación:

$$
\tau=\left(t_{2}-t_{1}\right)\left(1+\frac{a}{2 V}\left(t_{1}+t_{2}\right)\right)
$$

Considerando tiempos absolutos, $t_{1}=t_{1}^{\prime}$ y $t_{2}=$ $t_{2}^{\prime}$, encontramos de manera muy simple que la Ec. (5) nos entrega el siguiente resultado no-relativista, equivalente a la Ec. 2 , con $\tau=T^{\prime \prime}, \tau_{0}=T$ y $t_{1}+t_{2}=$ $2 t+T$.

$$
\tau=\tau_{0}+\frac{a}{2 V}\left(t_{1}+t_{2}\right) \tau_{0}
$$

Para obtener un resultado relativista, usamos las transformaciones de Lorentz y relacionamos los intervalos de tiempo en $\mathrm{S}$ y S' para eventos que ocurren en el origen de $\mathrm{S}$ ', encontrando $\gamma d t^{\prime}=d t$ y de manera aproximada para pequeños intervalos $\gamma \Delta t^{\prime}=\Delta t$. Con esta aproximación $t_{2}=t_{1}+\gamma \tau_{0}$ y es posible escribir la Ec 5 como:

$$
\tau=\frac{V+\frac{a \gamma \tau_{0}}{2}+a t}{V}\left(\gamma \tau_{0}\right)
$$

Esta expresión corresponde a la Ec. (4) intuída anteriormente, en el caso de una fuente acelerada que se aleja del observador 


\section{Discusión}

Los resultados obtenidos en la presente nota son pertinentes debido al hecho que han aparecido en la literatura de enseñanza de la física, experimentos que utilizan los teléfonos móviles inteligentes (smartphones) como acelerómetros [11-14]. Por otra parte, se ha descrito la extensión relativista de este fenómeno, mostrando que la simple sustitución $T \rightarrow \gamma T$ nos ofrece una buena aproximación al fenómeno relativista.

En resumen, el modo argumental usado en esta nota resalta la utilidad didáctica de los diagramas $(\mathrm{x}, \mathrm{t})$, cuya simpleza puede entregar resultados en temas que parecen didácticamente complejos. Como comentario final, nuestra opinión es que el efecto Doppler es un fenómeno simple de cinemática (ver p. ej., Neipp [9] y Bunn and Hogg [15]).

\section{Referencias}

[1] L. Fleischmann, American Journal of Physics 13, 418 (1945).

[2] V. Slüsarenko and C. H. Wörner The Physics Teacher 27, 171 (1989).

[3] L. Viennot and J.L. Leroy-Bury, Physics Education 39273 (2004)

[4] R. Rojas and G. Fuster The Physics Teacher 452 (2007).

[5] A. Roatta and R. Welti, Revista Brasileira do Ensino de Fisica 31, 1304 (2009).

[6] F. Wang The Physics Teacher 51, 154 (2013).

[7] R.A. Serway Physics for Scientist and Engineers ( $4^{\text {th }} E d$.) (Saunders College Publishing, Philadelphia, 1996), Chapter 17, Problem 40.

[8] A. Radovic Journal of Theoretics 5-4 (2003). Disponible en www.andrijar.com/doppler/dopplerfinal.pdf, (March, 2016).

[9] C Neipp, A. Hernández, J.J. Rodes, A. Márquez, T. Beléndez and A. Beléndez, European Journal of Physics 24, 497 (2003).

[10] J. Earman J and C. Glymour, Studies in History and Philosophy of Science Part A 11, 175 (1980).

[11] P. Vogt, J. Kuhn and S. Müller, The Physics Teacher, 49, 383 (2011).

[12] P. Vogt, J. Kuhn and S. Müller, The Physics Teacher 50, 182 (2012).

[13] J. Kuhn and P. Vogt European Journal of Physics Education 4, 16 (2013).

[14] J. Chevrier, L. Madani, S. Ledenmat and A. Bsiesy, The Physics Teacher 51, 376 (2013).

[15] E.F. Bunn and D.W. Hogg, Am. J. Physics 77, 688 (2009). 